\title{
Chronic pulmonary aspergillosis complicating sarcoidosis
}

\author{
Yurdagül Uzunhan ${ }^{1,2}$, Hilario Nunes ${ }^{1,2}$, Florence Jeny ${ }^{1,2}$, Maxime Lacroix ${ }^{1,3}$, \\ Sophie Brun ${ }^{4}$, Pierre-Yves Brillet ${ }^{1,3}$, Emmanuel Martinod ${ }^{5}$, \\ Marie-France Carette ${ }^{6}$, Diane Bouvry ${ }^{1,2}$, Caroline Charlier ${ }^{7,8}$, \\ Fanny Lanternier ${ }^{7,8}$, Carole Planès ${ }^{1,2}$, Abdellatif Tazi ${ }^{9}$, Olivier Lortholary ${ }^{7,8}$, \\ Robert P. Baughman ${ }^{10}$ and Dominique Valeyre ${ }^{1,2}$
}

\begin{abstract}
Affiliations: ${ }^{1}$ Paris 13 University, EA2363, Sorbonne Paris Cité, Bobigny, France. ${ }^{2}$ Assistance Publique Hôpitaux de Paris, Avicenne Hospital, Pulmonary Dept, Bobigny, France. ${ }^{3}$ Assistance Publique - Hôpitaux de Paris, Avicenne Hospital, Radiology Dept, Bobigny, France. ${ }^{4}$ Assistance Publique - Hôpitaux de Paris, Avicenne Hospital, Mycology and Parasitology Dept, Bobigny, France. ${ }^{5}$ Assistance Publique - Hôpitaux de Paris, Avicenne Hospital, Thoracic Surgery Dept, Bobigny, France. ${ }^{6}$ Assistance Publique - Hôpitaux de Paris, Tenon Hospital, Radiology Dept, Paris, France. ${ }^{7}$ Paris Descartes University, Sorbonne Paris Cité, Assistance Publique Hôpitaux de Paris, Necker-Enfants Malades Hospital, Necker Pasteur Center for Infectious Diseases and Tropical Medicine, Paris, France. ${ }^{8}$ Institut Pasteur, National Reference Center for Invasive Mycoses and Antifungals, CNRS URA3012, Paris, France. ${ }^{9}$ Paris Diderot University, Sorbonne Paris Cité, Assistance Publique - Hôpitaux de Paris, Saint-Louis Hospital, Pulmonary Dept, Paris, France. ${ }^{10}$ Interstitial Lung Disease and Sarcoidosis Clinic, Dept of Internal Medicine, University of Cincinnati, Cincinnati, $\mathrm{OH}$, USA.
\end{abstract}

Correspondence: Yurdagül Uzunhan, Hôpital Avicenne, 125 rue de Stalingrad, Bobigny, France. E-mail: yurdagul.uzunhandaphp.fr

@ERSpublications

CPA occurs in advanced pulmonary sarcoidosis, and survival is predicted by CPI, PH and fibrosis extent http://ow.ly/R3w830b2HvR

Cite this article as: Uzunhan Y, Nunes H, Jeny F, et al. Chronic pulmonary aspergillosis complicating sarcoidosis. Eur Respir J 2017; 49: 1602396 [https://doi.org/10.1183/13993003.02396-2016].

ABSTRACT Chronic pulmonary aspergillosis (CPA) complicating sarcoidosis (SA) is associated with high mortality, and there is a lack of clarity regarding the relative contributions of SA or CPA.

This was a retrospective single-centre study on CPA-SA.

In total, 65 patients (44 men), aged $41.4 \pm 13.5$ and $48.3 \pm 11.9$ years at the time of SA and CPA diagnoses, respectively, were included between 1980 and 2015. Of these, 64 had fibrocystic SA, most often advanced, with composite physiological index (CPI) $>40$ (65\% of patients) and pulmonary hypertension (PH) (31\%), and 41 patients (63\%) were treated for SA (corticosteroids or immunosuppressive drugs). Chronic cavitary pulmonary aspergillosis (CCPA) was the most frequent CPA pattern. Regarding treatment, 55 patients required long-term antifungals, 14 interventional radiology, 11 resection surgery and two transplantation. Nearly half of the patients $(27 ; 41.5 \%)$ died (mean age 55.8 years); $73 \%$ of the patients achieved 5-year survival and 61\% 10-year survival. Death most often resulted from advanced SA and rarely from haemoptysis. CPI, fibrosis extent and $\mathrm{PH}$ predicted survival. Comparison with paired healthy controls without CPA did not show any difference in survival, but a higher percentage of patients had high-risk mould exposure.

CPA occurs in advanced pulmonary SA. CPA-SA is associated with high mortality due to the underlying advanced SA rather than to the CPA. CPI, fibrosis extent and PH best predict outcome.

This article has supplementary material available from erj.ersjournals.com

Received: July 112016 | Accepted after revision: Feb 182017

Conflict of interest: Disclosures can be found alongside this article at erj.ersjournals.com

Copyright OERS 2017 


\section{Introduction}

Chronic pulmonary aspergillosis (CPA) may complicate fibrocystic pulmonary sarcoidosis $(\mathrm{SA})[1,2]$. The burden of CPA-SA has been estimated at 3-12\% of cases [2], and SA might be the underlying predisposing condition for CPA in $7.1 \%$ of cases [3]. In the literature, CPA-SA is reported to have a high mortality rate [4-13], with no CPA categorisation and no clear information on the respective contributions of CPA and SA to death [2]. Studies have implicated corticosteroid and immunosuppressive treatments in CPA-SA, as well as underlying pulmonary fibrocystic lesions and smoking habit [11].

We report a series of 65 patients with CPA-SA seen in a tertiary centre for SA, who were monitored with the support of a French infectious disease reference centre for invasive mycoses. Comparisons were made with a healthy control (HC) group with a similar phenotype except for CPA. Our goals were to: 1) investigate CPA categorisation and SA phenotyping at diagnosis, 2) look for CPA-SA risk factors, 3) describe the effect of antifungals on CPA outcome, 4) assess survival, 5) specify the respective contribution of CPA and SA on mortality, and 6) look for predictive outcome factors.

\section{Methods}

This retrospective study was conducted in Avicenne Hospital Pulmonology Dept with institutional review board approval (CLEA-R13).

\section{Patient selection}

The database of patients admitted to the pulmonary department was examined. SA and CPA diagnoses were matched for the 1980-2015 period. The included patients met the following criteria. SA diagnosis made according to the 1999 statement on SA [14]. Evidence of noncaseating granulomas was required. CPA diagnosis based on the presence of: 1) at least one pulmonary cavity on thoracic imaging with or without one or more fungal balls in a cavity, and 2) positive blood anti-Aspergillus immunoglobulin $G$ antibodies or 3) cultures/histology implicating Aspergillus spp. [1] or showing hyphae compatible with Aspergillus.

The HC group was made up of patients with fibrocystic SA without current or subsequent CPA treated in the same department, paired 1:1 with the patient group according to the date of the fibrocystic lung diagnosis (difference $<5$ years).

\section{Clinical, pulmonary function, haemodynamics and biological characteristics at CPA diagnosis}

Entry into the study was the date of CPA diagnosis. The following information was collected: age, sex, geographical origin, smoking habit, high-risk occupational exposure to air moulds and dusts, and presence of comorbid or past conditions. Extrapulmonary manifestations were recorded [15].

Pulmonary function tests included spirometry $(\mathrm{n}=64)$, diffusing capacity of the lung for carbon monoxide (DLCO) $(\mathrm{n}=64)$ and room-air blood gases $(\mathrm{n}=42)$. Forced vital capacity (FVC), forced expiratory volume in $1 \mathrm{~s}(\mathrm{FEV} 1)$ and DLCO were expressed as a percentage of predicted values $[16,17]$.

Doppler echocardiography was performed on all patients within 6 months of CPA diagnosis. Pulmonary hypertension $(\mathrm{PH})$ was defined by estimated systolic pulmonary artery pressure $>40 \mathrm{mmHg}$.

Available serum C-reactive protein $(\mathrm{n}=35)$ and serum angiotensin-converting enzyme (sACE) ( $\mathrm{n}=48)$ levels were recorded.

\section{Computed tomography analysis}

For further information on the computed tomography analysis, see the supplementary material. Computed tomography scans were reviewed in concert by two authors (PYB, a radiologist with 15 years of experience in interstitial lung disease, and YU) at CPA diagnosis.

Fibrotic lesions were classified into four patterns according to ABESEHra et al. [18]. Total disease extent was rounded to the nearest $5 \%$, and the relative proportions of the individual patterns contributing to the total disease extent were estimated to the nearest 5\%. Main pulmonary artery diameter (MPAD) and ascending aorta diameter (AAD) had been assessed in high-resolution computed tomography (HRCT) scans at CPA diagnosis.

CPA categorisation was applied according to Denning et al. [1].

\section{Composite physiological index and Walsh's algorithm}

The composite physiological index (CPI) was calculated [19] and the prognosis algorithm of WALSH et al. [20] applied. 


\section{Fungal investigations}

For further information on the fungal investigations, see the supplementary material. Tests for serum Aspergillus-specific IgG were carried out for 64 patients. Fungal culture was performed on sputum $(\mathrm{n}=47)$ or bronchoscopy aspirate $(n=44)$.

\section{Risk factors for CPA}

We looked for risk factors for CPA by comparing the CPA-SA and HC groups. Attention was paid to geographical origin, smoking habit, previous pneumothorax, SA treatments and the presence of diabetes mellitus at time of diagnosis of fibrocystic SA.

Patients' mould exposure was also assessed (patients' employment was mentioned in the files). We considered exposures and job titles that have been associated with aspergillosis in prior studies [21]; construction work, farming, gardening, florist shop work and forestry work were considered high-risk jobs for fungal spores [22].

\section{Treatments and outcome}

Patients were monitored through regular visits and tests at time intervals depending on clinical requirements. The physician in charge prescribed treatment. Every event was recorded, and we looked for factors predictive of survival. Survival in the CPA-SA group was compared with the HC group.

For the CPA follow-up evaluation, we compared CT before and after 6-12 months of antifungals using the criteria of Godet et al. [23]. The following data were collected: number and volume of cavities, number and maximal diameter of fungus balls, maximal thickness of cavity wall and maximal pleural thickening. Maximal diameter of the largest cavities, alveolar consolidation, lobar collapse, ground-glass attenuation and nodules were also assessed. According to GoDET et al. [23], maximal thickness of cavity wall and maximal pleural thickening are the most relevant CT variants for assessing response to treatment.

\section{Statistical analysis}

Quantitative values are reported as mean and SDS, and qualitative data as numbers (percentages). Information regarding vital status and causes of death were obtained by reviewing medical records and by contacting referral physicians or general practitioners. Survival was calculated from inclusion, i.e. date of CPA diagnosis until the end of the follow-up period.

We used STATA software (version 13; StataCorp, College Station, Texas, USA) for statistical analyses. We performed group comparisons using the $t$-test, Wilcoxon rank sum, Chi-squared statistics and Fisher's exact test where relevant. Univariate analysis based on the proportional hazards model was used with the log-rank test. All significant parameters were entered into the multivariate Cox proportional hazards model. Survival curves were based on the Kaplan-Meier method and compared using the log-rank test. For all statistical analyses, $\mathrm{p}=0.05$ was considered significant.

\section{Results}

Selection of patients

From our database of 3137 patients with SA, 80 patients with pulmonary CPA-SA recorded between January 1980 and December 2015 were identified; of these, 15 patients were excluded (insufficient medical data $(n=5)$, no confirmed SA $(n=4)$, incomplete CPA criteria $(n=6))$. The remaining 65 patients $(2 \%$ of the SA population) made up the final study population.

The HC group (64 patients) was similar to the CPA-SA group in age, FVC, FEV1, DLCO and CPI (figure 1 and table 1).

\section{Presentation at CPA diagnosis}

\section{Population characteristics}

The population characteristics are summarised in table 2. The diagnosis of SA preceded the diagnosis of CPA for 55 patients (85\%), while CPA and SA diagnoses were made concomitantly in 10 cases (15\%).

\section{CPA presentation}

Of the 65 patients, 24 (37\%) presented haemoptysis at CPA diagnosis, 26 (40\%) had weight loss (mean $3.5 \mathrm{~kg}$ ) and $29(45 \%)$ reported fatigue. CPA presentation could be categorised in most patients (figure 2). The most frequent phenotypes were chronic cavitary pulmonary aspergillosis (CCPA) $(n=27)$ and simple aspergilloma $(\mathrm{n}=17)$. There was one case of subacute invasive aspergillosis (SAIA). Some overlaps between CCPA and aspergillomas or between aspergillomas and chronic fibrosing pulmonary aspergillosis and sometimes between all three entities were noted, while some phenotypes were not classifiable due to confusing SA lesions $(\mathrm{n}=10)$. The biological data concerning CPA diagnosis are summarised in table 3 . In 


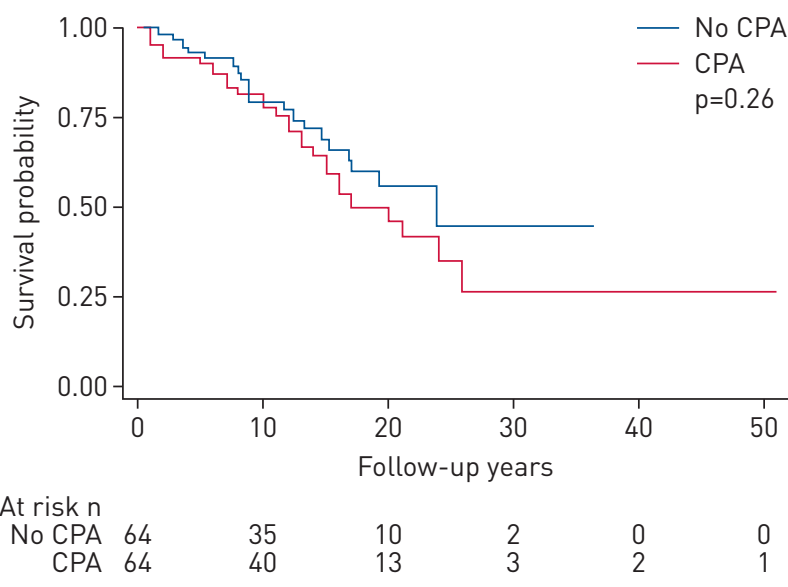

FIGURE 1 Survival of patients after fibrocystic lung sarcoidosis diagnosis with and without cavitary pulmonary aspergillosis (CPA) paired according to the date of stage 4 diagnosis (difference $<5$ years).

92.3\% of patients, Aspergillus-specific IgG was found, and in 77.9\%, Aspergillus was found in endobronchial and/or sputum specimens. Two patients from the same family developed allergic bronchopulmonary aspergillosis (ABPA): 5 years after CPA for one and concomitantly for the other. Both had hypereosinophilia, high IgE levels, evidence of Aspergillus-specific IgE and positive Aspergillus prick tests.

\section{SA manifestations}

There were 64 patients with fibrocystic pulmonary SA at CPA diagnosis. The main CT fibrosis pattern was bronchial distortion, often with pulmonary masses (table 2, figures 2 and 3). Regarding HRCT findings, SA was mildly active in 49 cases (83.1\%). sACE was elevated in $42 \%$ of patients (table 3 ). Two-thirds of patients received systemic treatment for SA prior to $(n=44 ; 67.7 \%)$ or at CPA diagnosis $(n=41 ; 63.0 \%)$. Pulmonary disease was often severe, as judged by CPI score $>40(n=42,65 \%), P H(n=20,31 \%)$, poor

TABLE 1 Characteristics of patients after fibrocystic lung sarcoidosis diagnosis with and without cavitary pulmonary aspergillosis paired according to the date of stage 4 diagnosis (difference $<5$ years)

\begin{tabular}{lccc} 
& Cases & Controls & p-value \\
\hline Subjects n & 64 & 64 & \\
Male & $43(67.1 \%)$ & $32(50 \%)$ & 0.07 \\
SS or AC & $21(32.8 \%)$ & $21(32.8 \%)$ & 1 \\
Age years at stage 4 & $43 \pm 13.5$ & $41.8 \pm 9.5$ & 0.66 \\
Smoking NS/ES (mean pack-years) & $43(67.1 \%) / 22(34.3 \%)(5.5)$ & $37(57.8 \%) / 27(42.1 \%)(5.7)$ & 0.36 \\
Diabetes mellitus & $7(10.9 \%)$ & $6(9.3 \%)$ & 1 \\
Pneumothorax & $8(12.5 \%)$ & $5(7.8 \%)$ & 0.56 \\
High-risk occupational exposure & $24(37.5 \%)$ & $11(17.1 \%)$ & 0.01 \\
PFT at stage 4 diagnosis & & & 0.58 \\
FEV $\%$ predicted & $58.7 \pm 18.8$ & $61.4 \pm 22.2$ & 0.06 \\
FVC \% predicted & $62.2 \pm 18.6$ & $69.6 \pm 22.8$ & 0.35 \\
DLCO \% predicted & $50.7 \pm 16.7$ & $53.3 \pm 17.0$ & 0.07 \\
CPI & $45.5 \pm 14.8$ & $39.7 \pm 16.2$ & 0.29 \\
Treatment for sarcoidosis at stage & $53(82.8 \%)$ & $58(90.6 \%)$ & 0.83 \\
$\quad \mathbf{4}$ diagnosis & & $15(23.4 \%)$ & 1 \\
CTS $\leqslant 10$ mg per day & $13(20.3 \%)$ & $33(51.5 \%)$ & 0.79 \\
CTS $>10$ mg per day & $32(50 \%)$ & $10(15.6 \%)$ & \\
Immunosuppressive drugs & $8(12.5 \%)$ & & \\
\hline
\end{tabular}

Data are presented as $\mathrm{n}(\%)$ or mean \pm SD unless otherwise stated. SS: sub-Saharan origin; AC: Afro-Caribbean origin; NS: never-smoker; ES: ever-smoker; PFT: pulmonary function test; FEV1: forced expiratory volume in $1 \mathrm{~s}$; FVC: forced vital capacity; DLCO: diffusing capacity of the lung for carbon monoxide; CPI: composite physiologic index $(91.0-(0.65 \times D \mathrm{LCO})-(0.53 \times \mathrm{FVC})+(0.34 \times \mathrm{FEV} 1)$; CTS: corticosteroids. 
TABLE 2 Patient characteristics at diagnosis of chronic pulmonary aspergillosis (CPA) ( $n=65$ )

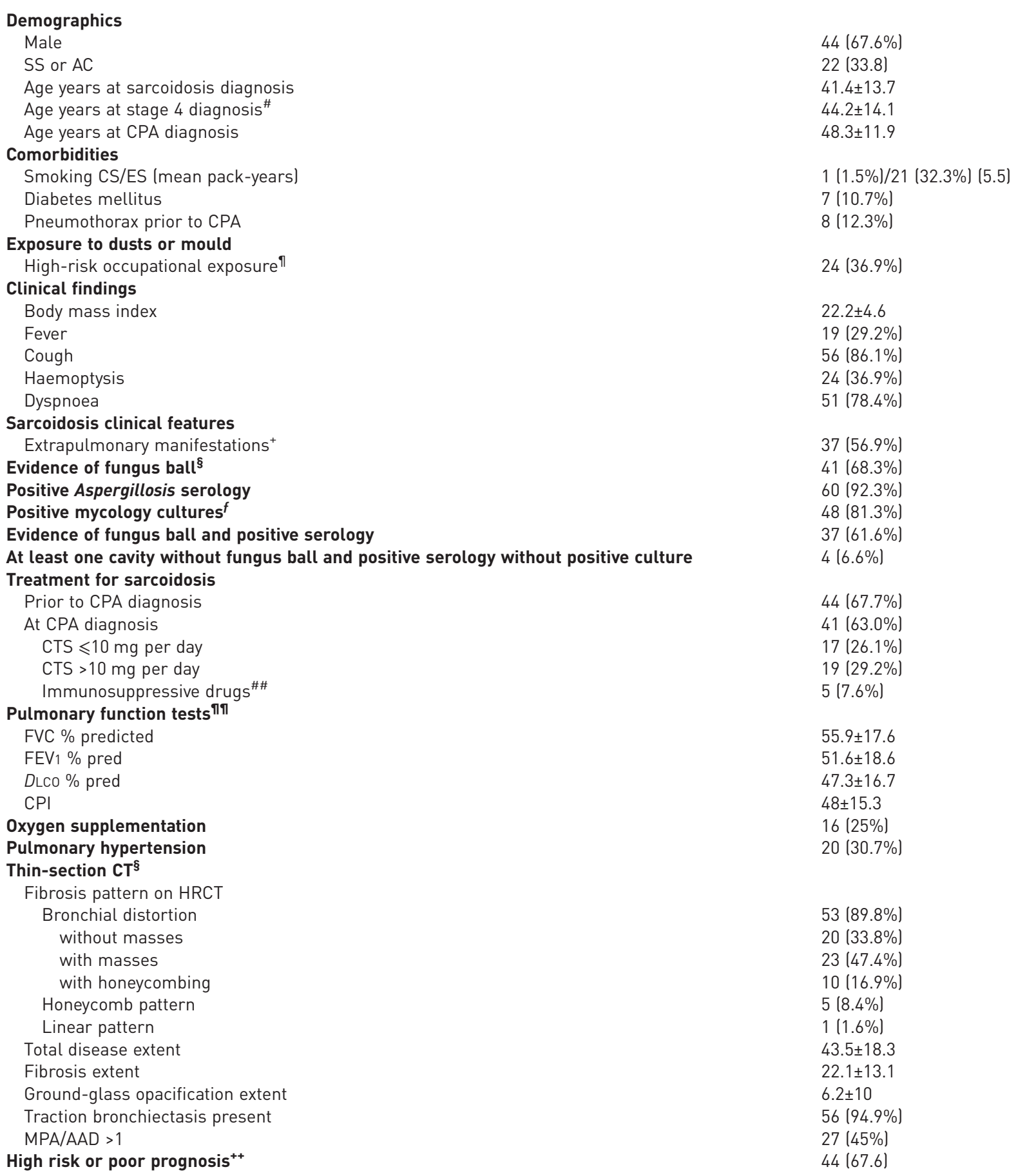

Data are presented as $\mathrm{n}(\%)$ or mean \pm SD. SS: sub-Saharan origin; AC: Afro-Caribbean origin; CS: current smoker; ES: ever-smoker; CTS: corticosteroids; FVC: forced vital capacity; FEV1: forced expiratory volume in $1 \mathrm{~s}$; DLCo: diffusing capacity of the lung for carbon monoxide; CPI: composite physiologic index $(91.0-(0.65 \times D L C 0)-(0.53 \times F V C)+(0.34 \times F E V 1)$; CT: computed tomography; HRCT: high-resolution computed tomography; MPA/AAD: main pulmonary artery/ascending aorta diameter ratio. \#: all patients but one (stage 3 ) had stage 4 sarcoidosis at CPA diagnosis; 17 patients $(26.2 \%)$ presented with other stages at sarcoidosis onset, with three being stage $1(7.6 \%), 10$ stage $2(15.3 \%)$ and four stage $3(6.1 \%)$. ๆ: patients with CPA had worked in the following job categories: house-building ( $n=12)$, mechanics ( $n=3)$, electrics $(n=3)$, agriculture $(n=2)$, locksmithing ( $n=2)$ and carpentry $(n=2)$, all considered high-risk jobs according to SIPSAS et al. [22]. ${ }^{+}$: according to JUDSON et al. [15]. §: data available for 60 patients. $f$ : data available for 59 patients. \#\#: immunosuppressive drugs were azathioprine (n=4) or methotrexate $(n=1)$. กा: pulmonary disease was quite severe, with impairment in pulmonary function tests in the majority of cases, with restrictive pattern for 29 (44.6\%), obstructive pattern for nine (13.8\%) and mixed pattern for 20 patients (30.7\%) at CPA diagnosis. ${ }^{++}$: according to the algorithm proposed by WALSH et al. [20]. 


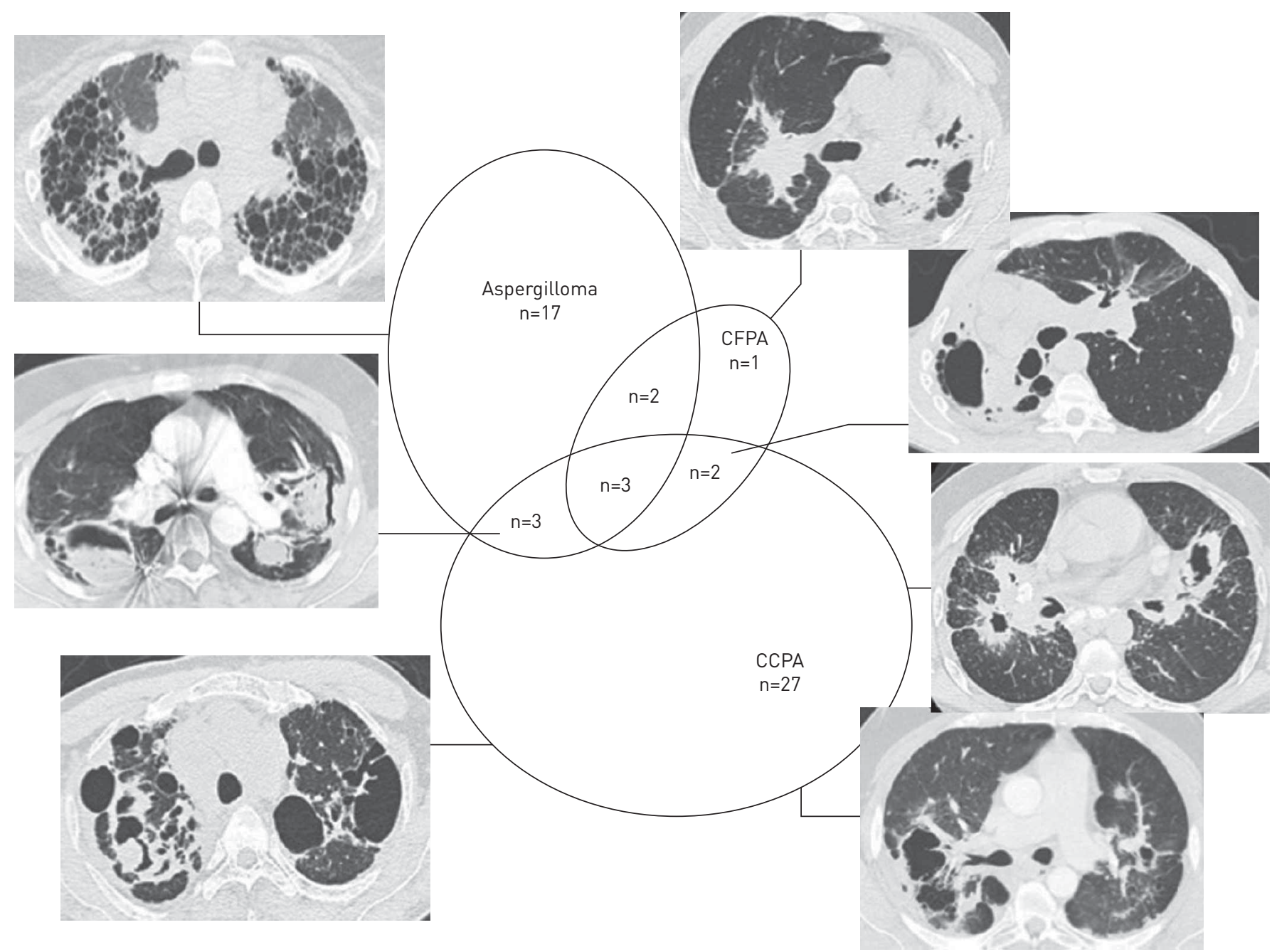

FIGURE 2 Chronic pulmonary aspergillosis classification on high-resolution computed tomography imaging at diagnosis. CCPA: chronic cavitary pulmonary aspergillosis; CFPA: chronic fibrosing pulmonary aspergillosis. One case of subacute invasive aspergillosis not shown on this figure.

\begin{tabular}{|c|c|}
\hline \multicolumn{2}{|l|}{ Laboratory data } \\
\hline Elevated serum ACE (fold increase over normal) & $19(41.6 \%)(1.32 \pm 0.59)$ \\
\hline C-reactive protein $\left(\mathrm{mg} \cdot \mathrm{L}^{-1}\right)^{\Uparrow}$ & $27(87 \%)(49.9 \pm 67.1)$ \\
\hline Positive aspergillosis serology & $60(92.3 \%)$ \\
\hline \multicolumn{2}{|l|}{ Microbiological data } \\
\hline \multicolumn{2}{|l|}{ Mycology ${ }^{+}$} \\
\hline All $n$ & 59 \\
\hline A. fumigatus & $46(77.9 \%)$ \\
\hline A. nidulans & $2(3.3 \%)$ \\
\hline A. fumigatus $+A$. flavus & $1(1.6 \%)$ \\
\hline A. fumigatus $+A$. niger & $1(1.6 \%)$ \\
\hline A. fumigatus $+A$. nidulans & $1(1.6 \%)$ \\
\hline Antifungal susceptibility testing $n$ & 19 \\
\hline Positive Aspergillus antigen in blood $n / n$ & $9 / 34$ \\
\hline Co-infections & $30(46.1 \%)$ \\
\hline Bacterial infections $\S^{\S}$ & $19(29.2 \%)$ \\
\hline Mycobacterial infections ${ }^{f}$ & $3(4.6 \%)$ \\
\hline \multicolumn{2}{|c|}{ 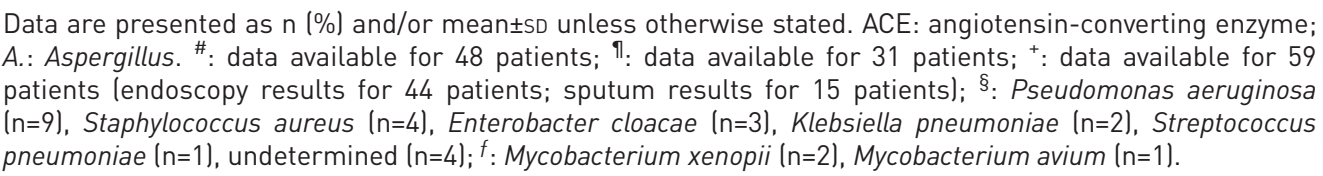 } \\
\hline
\end{tabular}



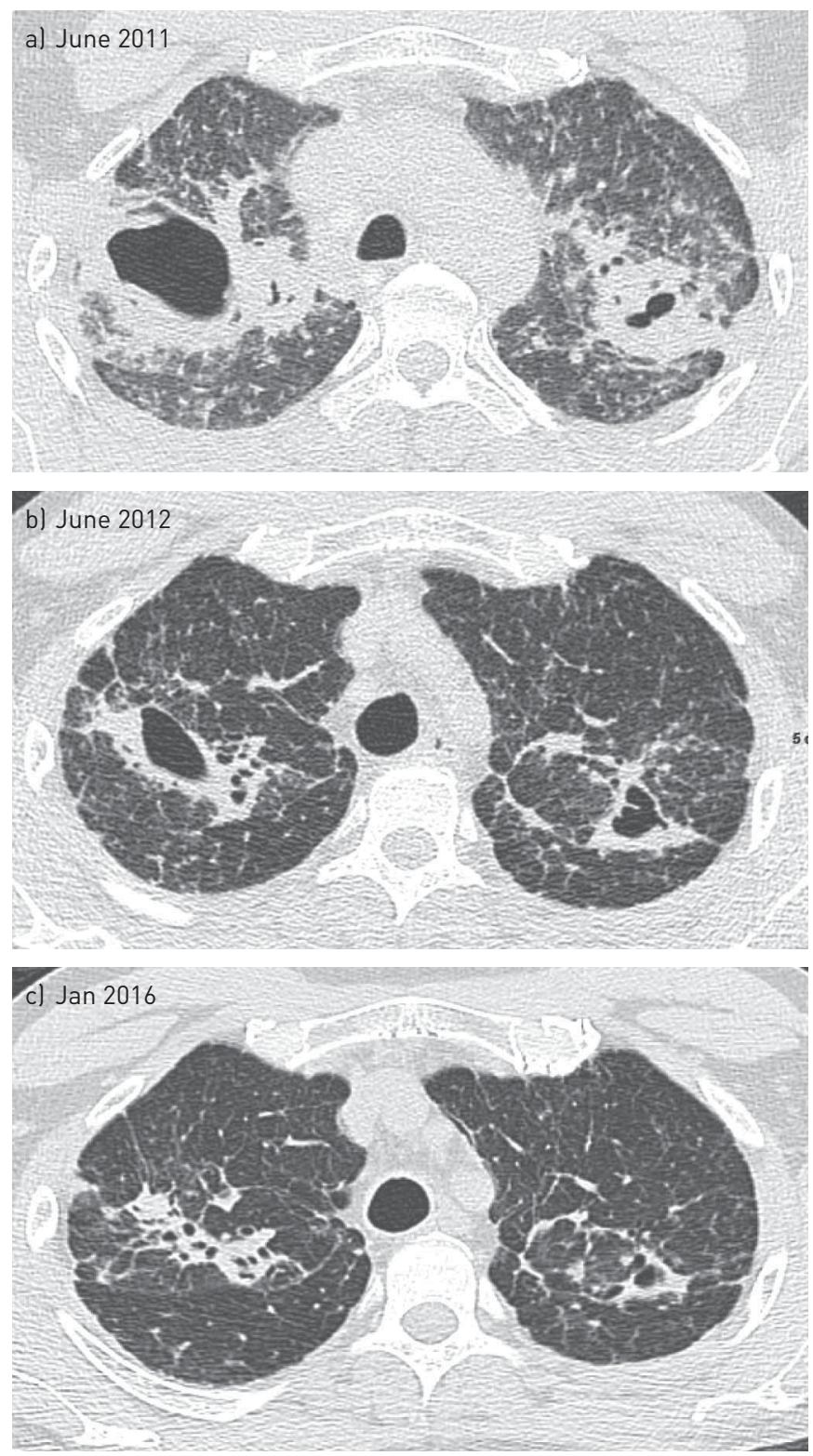

FIGURE 3 Computed tomography images of one patient with cavitary pulmonary aspergillosis and sarcoidosis, showing a distortion bronchial fibrosis pattern and excavated masses; the patient was successfully treated by voriconazole and strengthening of corticosteroid treatment. This figure shows the decrease in the cavity wall thickness under treatment according to the criteria of GoDEt et al. [23].

survival prognosis according to the algorithm of WALSH et al. [20] ( $\mathrm{n}=44,66 \%)$, or oxygen supplementation $(\mathrm{n}=16,25 \%)$ (table 2$)$.

\section{CPA risk factors}

There was no difference between the CPA-SA and HC groups in geographical origin, smoking habit, pneumothorax in the past, diabetes or treatment for SA (figure 1 and table 1). There was a trend towards a higher proportion of males in the CPA population, and the proportion of high-risk mould-exposure jobs was significantly higher in the CPA-SA than in the HC group (figure 1 and table 1).

\section{CPA treatment and outcome}

At CPA diagnosis, besides receiving treatment targeting SA, patients received oxygen supplementation $(\mathrm{n}=16,25 \%)$ and drugs to treat pulmonary hypertension $(\mathrm{n}=6,9 \%)$.

During the course of CPA, patients were treated for CPA or haemoptysis. Major haemoptysis was considered to be due mainly to CPA (figure 4). However, haemoptysis might have been due to 

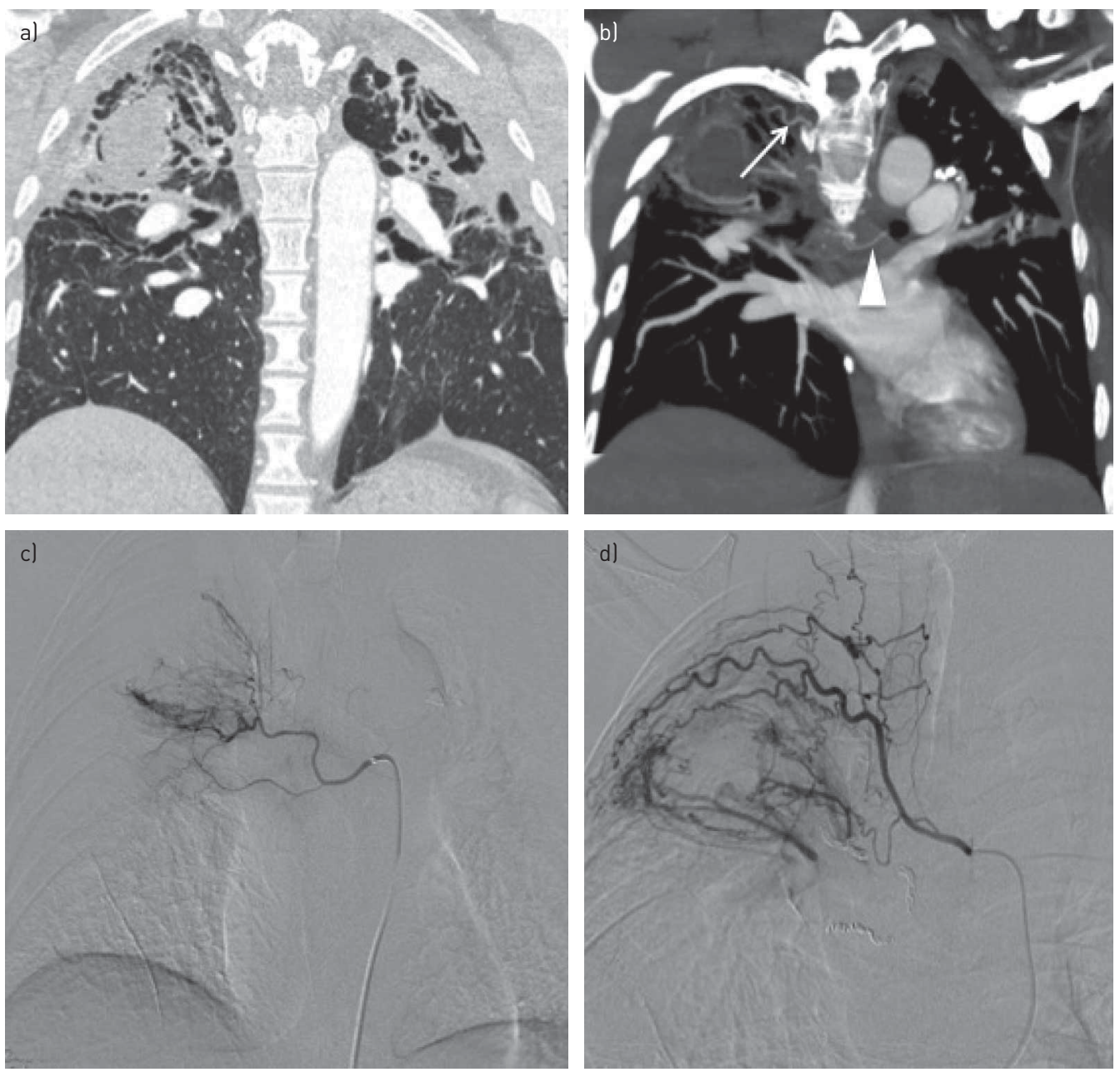

FIGURE 4 Angio-computed tomography images with coronal display in the a) parenchymal and b) mediastinal windows, showing cavitation in both upper lobes with a large aspergilloma in the right upper lobe cavity. The main hypervascularisation comes from the bronchial artery (arrowhead) and intercostal artery (arrow). Bronchial dilatation is also visible under the right aspergilloma (a). Systemic arteriography showing the c) right bronchointercostal trunk with marked hypertrophia of the third intercostal artery around the excavation due to sarcoidosis with cavitary pulmonary aspergillosis, d) without any arterial hypertrophy around the bronchiectasis and right upper bronchial artery, responsible for a hypervascularisation mainly at the bottom of the cavity.

bronchiectasis, which was quasi-constant or boosted by bacterial co-infections, suspected in $46 \%$ of patients with a bacterial infection and confirmed for 29 patients, or to non-tuberculous mycobacterial infections in three patients (4.6\%) (table 3$)$.

In total, 21 bronchial arterio-embolisations (BAEs) were performed for abundant or recurrent haemoptysis in 14 patients, 11 of whom had underlying CCPA (78.5\%). Nonlethal severe adverse events of BAE were observed in four cases: stroke $(n=2)$, bronchial arterial dissection $(n=1)$ and haemomediastinum $(n=1)$.

11 patients underwent resection surgery for CPA. The main indication was severe haemoptysis $(n=8)$, while one patient needed the surgery before being listed for renal transplantation, another had surgery for persistent pneumothorax and the last patient was cured after resection of a single excavated lesion. The procedures included lobectomy $(n=4)$, sublobar resection $(n=5)$, segmentectomy $(n=4)$, decortication $(n=2)$, pleurectomy $(n=1)$ and thoracoplasty $(n=1)$. There were no peri-operative deaths within 30 days. Nine of the 11 patients received antifungal treatment before and for the 3 months following surgery, and 10 patients had a recurrent CPA after surgery with a median delay of 12 months (range 6-45 months). Four patients (36\%) died during a longer follow-up. Survival was not significantly linked to surgery $(p=0.48)$.

Nine patients had been considered for lung transplantation; seven were rejected for unresolved aspergillosis, and the remaining two patients received lung transplant. At the time of both transplantations, aspergillosis serology titre was low and there was no mycological complication. One 
patient died after 24 months because of chronic graft dysfunction, while the other was still alive 8 years after transplantation.

Fifty-five patients received antifungal treatments for more than 6 weeks. Antifungal treatment relied on azoles, mostly on voriconazole $(n=38)$ and itraconazole $(n=23)$ (table 4). Serum concentration was monitored for 24 patients during azole therapy. Because of adverse effects (mostly liver toxicity with cytolysis (20\%) and voriconazole-associated phototoxicity (7.2\%)), these drugs were discontinued for one-third of patients. The treatment was withdrawn after good clinical/radiological response, at least $6-$ 12 months later, but resumed later, possibly because of a new CPA flare-up. Corticosteroids were reduced during CPA flare-up and increased again during SA exacerbation. The case presented in figure 3 illustrates the result obtained by voriconazole followed by a stronger SA treatment, allowing both control of CPA and reduction of the underlying SA lesions. One patient with ABPA responded well to voriconazole. Cough and general symptoms often improved with antifungals. The effect of antifungals (voriconazole in 24 patients and itraconazole in 6) could be assessed in 30 patients who underwent antifungal therapy for at least 6 months, by comparison of CT scans taken before and after 6-12 months of treatment (table 5). The maximal thickness of the cavitation wall $(\mathrm{p}=0.007)$ and pleura $(\mathrm{p}=0.02)$ decreased significantly.

\section{Survival, mechanisms of death and predictive outcome factors}

Survival

Patients were followed up for 7.5 \pm 6.2 years; $73 \%$ achieved 5 -year and $61 \%$ achieved 10 -year survival (figure 5). At the end of the study, 37 patients were alive (two of whom had undergone lung transplantation), one patient was lost to follow-up and 27 (41.5\%) were dead. Mean age at death was 55.8 years (range 3179 years). Survival did not differ with date of CPA diagnosis (before or after 2000). Survival from the date of diagnosis of fibrocystic lesions was not different between patients with CPA-SA and HCs $(p=0.26)$ (figure 1 and table 1).

\section{Causes of death}

For two patients, the cause of death was not ascertained. Respiratory failure was the most common cause of death $(\mathrm{n}=13 ; 48.1 \%)$. Nine patients died from right heart failure $(33.3 \%)$ and three died from severe haemoptysis $(11.1 \%)$. Death in these three cases was probably due to CPA, as the review of bronchial arteriography in nine out of 14 patients who underwent BAE found bronchial hypertrophy in the area of cavities related to CPA in all cases (figure 4). For 22 patients (34\% of the total; $81 \%$ of patients who died), death was attributable to the underlying advanced SA alone.

\section{Survival prediction factors}

As shown in table 6, the following parameters at CPA diagnosis were significantly associated with a higher death risk on univariate analysis: age $(\mathrm{p}=0.002)$, FVC $(\mathrm{p}=0.003)$, DLCO $(\mathrm{p}=0.003)$, CPI $>40(\mathrm{p}=0.015)$, CT fibrosis extent $(p=0.001)$, MPAD/AAD ratio on HRCT $(p=0.05)$, pulmonary hypertension $(p=0.001)$, Walsh algorithm $(p=0.04)$ and diabetes $(p=0.016)$. The independent predictive indicators of mortality at CPA diagnosis were $\mathrm{PH}(\mathrm{p}=0.003)$, CPI $(\mathrm{p}=0.004)$ and fibrosis extent $(\mathrm{p}=0.002)$ (figure 5).

\section{Discussion}

Our study reports the largest cohort on CPA-SA to date, and provides several answers to important unsolved questions: 1) advanced pulmonary fibrocystic SA, often with a bronchial distortion pattern associated with masses, was almost always an underlying basis for CPA-SA; 2) a job with high-risk mould

\begin{tabular}{lc}
$\begin{array}{l}\text { TABLE } 4 \text { Antifungal treatment given to patients with chronic pulmonary aspergillosis } \\
\text { complicating sarcoidosis }\end{array}$ & Patients \\
\hline Treatment & $55(84.6 \%)$ \\
\hline All & $27(41.5 \%)$ \\
Voriconazole & $15(23 \%)$ \\
Itraconazole & $4(6.1 \%)$ \\
Itraconazole followed by voriconazole & $4(6.1 \%)$ \\
Voriconazole followed by itraconazole & $3(4.6 \%)$ \\
Voriconazole followed by posaconazole & $1(1.5 \%)$ \\
Intravenous amphotericin B & $2(3 \%)$ \\
Caspofungin & $1(1.5 \%)$ \\
Nebulised liposomal amphotericin B &
\end{tabular}


TABLE 5 Computed tomography findings of patients before and 6-12 months after antifungal treatment $^{\#}$

\begin{tabular}{|c|c|c|c|}
\hline & Before treatment & After treatment & p-value \\
\hline Patients n & 30 & 30 & \\
\hline Presence of at least one cavity & $30(100 \%)$ & $30(100 \%)$ & \\
\hline Unilateral localisation of cavity & $9(30 \%)$ & $10(33.3 \%)$ & \\
\hline \multicolumn{4}{|l|}{ Cavities in the right lung } \\
\hline 0 & $4(13.3 \%)$ & $4(13.3 \%)$ & \\
\hline $1-5$ & $22(73.3 \%)$ & $21(70 \%)$ & \\
\hline $5-10$ & $1(3.3 \%)$ & $2(6.6 \%)$ & \\
\hline$>10$ & $3(10 \%)$ & $3(10 \%)$ & \\
\hline \multicolumn{4}{|l|}{ Cavities in the left lung } \\
\hline None & $6(20 \%)$ & $6(20 \%)$ & \\
\hline $1-5$ & $17(56.6 \%)$ & $18(60 \%)$ & \\
\hline $5-10$ & $4(13.3 \%)$ & $3(10 \%)$ & \\
\hline$>10$ & $3(10 \%)$ & $3(10 \%)$ & \\
\hline Average diameter of largest cavities $\mathrm{mm}$ & $28.5 \pm 17.8$ & $23.4 \pm 11.4$ & 0.12 \\
\hline Maximal diameter of infected cavities $\mathrm{mm}$ & $37.6 \pm 20.4$ & $28.4 \pm 14.1$ & 0.059 \\
\hline Presence of fungus balls & $21(70 \%)$ & $18(60 \%)$ & 0.27 \\
\hline Unilateral localisation of fungus balls & $15(50 \%)$ & $13(43.3 \%)$ & 0.79 \\
\hline Maximal diameter of fungus ball $\mathrm{mm}$ & $16.7 \pm 11.0$ & $16.0 \pm 10.8$ & 0.84 \\
\hline Presence of nodules $>10 \mathrm{~mm}$ & $2(6.6 \%)$ & $2(6.6 \%)$ & \\
\hline Presence of ground-glass attenuation & $7(23.3 \%)$ & $7(23.3 \%)$ & \\
\hline Presence of areas of consolidation & $6(20 \%)$ & $7(23.3 \%)$ & \\
\hline Presence of lobar collapse & $3(10 \%)$ & $3(10 \%)$ & \\
\hline Maximum thickness of pleura mm & $8.2 \pm 3.6$ & $5.8 \pm 4.0$ & 0.02 \\
\hline Maximum thickness of cavity wall $\mathrm{mm}$ & $5.6 \pm 2.6$ & $3.6 \pm 1.8$ & 0.007 \\
\hline
\end{tabular}

exposure was a significant risk for CPA occurrence, irrespective of patient's geographical origin, diabetes, smoking habit, previous pneumothorax and treatment of SA; 3) CPA did not significantly affect survival, and mortality was high with 5- and 10 -year survival rates of $73.7 \%$ and $61 \%$, respectively; 4) mortality was mainly related to advanced pulmonary SA, while at most three out of 27 deaths were attributable to CPA through massive haemoptysis; 5) CPI, PH and pulmonary fibrosis extent (as assessed by CT) predicted survival; and 6) antifungals improved patients' condition in the short term.

There is only limited information in the literature on CPA-SA, which is often dated, with few cases, rare survival data and no CPA categorisation. The only survival study is that of TOMLINSON and SAHN on 14 patients with $58 \%$ deaths at 2 years [8], while DenNing et al. suggested a lower annual attrition rate with a range of $10-25 \%$ [2]. Combining four series showed 44 deaths out of 93 patients, i.e. 47\% [5-8]; 12 of the 44 deceased patients (27\%) died from massive haemoptysis [5-8]. Corticosteroid treatments, smoking history or having previous pneumothorax were suspected of being the highest risk factors for CPA. Spontaneous aspergilloma resolution is a very rare outcome $[7,9,24]$, while successful surgical resections [6] and individual benefits of interventional radiology [5, 8], systemic antifungals [25] and perendoscopic or transcutaneous instillation of fungicides $[26,30]$ have all been reported.

An advanced underlying fibrocystic lung disease was clearly shown to be the predisposing ground to CPA occurrence in SA. Masses, often associated with SA, secondarily excavated, might be the nidus for CPA. Moreover, $66 \%$ of patients met the criteria for poor survival prognosis [20]. Survival in patients with CPA-SA was not different from that of HCs, and $81 \%$ of deaths were due to respiratory insufficiency or cor pulmonale. Survival prediction factors (CPI, PH and fibrosis extent) were linked with advanced pulmonary SA, making our study an external validation cohort for the findings of WALSH et al. [20] concerning survival prediction criteria in advanced pulmonary SA. The absence of extra mortality due to CPA has also been observed in ancient series that had significant underlying tuberculosis sequels as the underlying condition [24]. Lower doses of corticosteroids and immunosuppressive treatments following CPA flare-up, and use of antifungals and interventional radiology might explain the reduction in mortality in our series compared with that of TomLinson et al. [8], even though our study can only presume such interpretation.

CCPA was the most frequent presentation in our SA series. In $10 \%$ of patients, classifying CPA was impossible because of the presence of confusing SA lesions. SA should be added as a cause of ABPA, as 


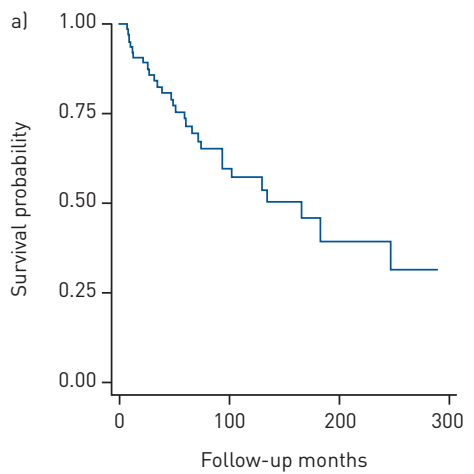

At risk $\mathrm{n}$ b)

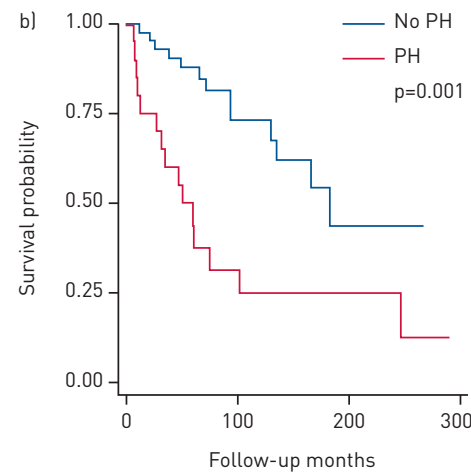

At risk $n$

No PH 45

$\mathrm{PH} 20$

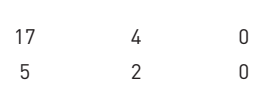

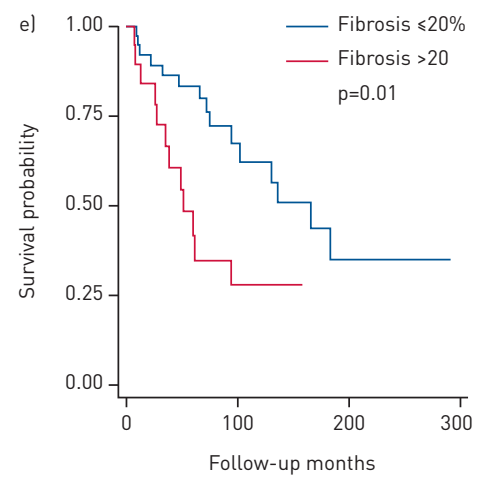

At risk $\mathrm{n}$

Fibrosis $\leqslant 20 \% \quad 39$

Fibrosis $>20 \% \quad 19$
14

3

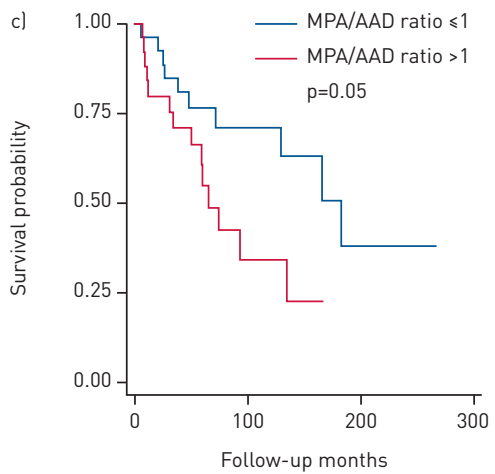

At risk $n$

MPA/AAD ratio $\leqslant 1 \quad 28 \quad 11 \quad 3 \quad 0$

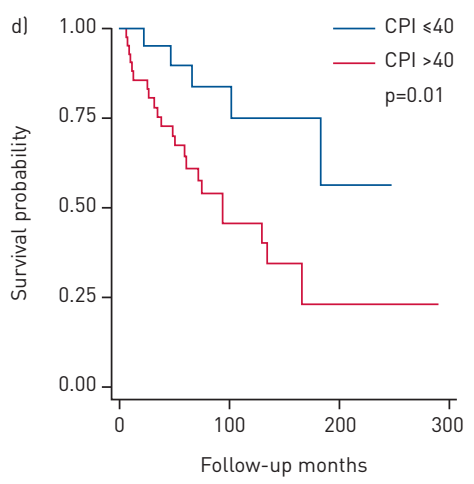

At risk $\mathrm{n}$

$\mathrm{CPI} \leqslant 4042 \quad 10 \quad 2 \quad 0$

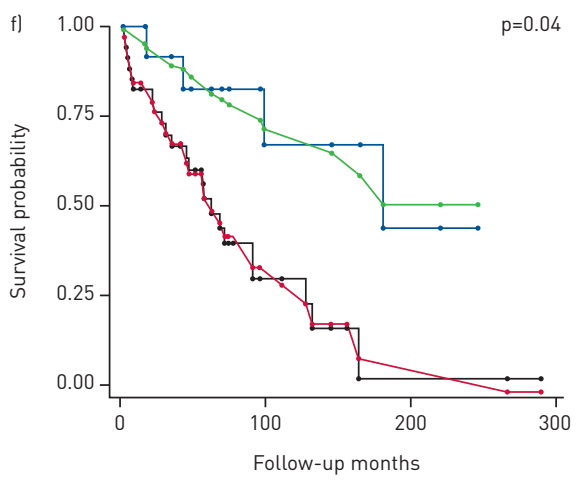

- Observed: good prognosis _ Observed: poor prognosis _ Predicted: bad prognosis _ Predicted: poor prognosis

FIGURE 5 Survival after cavitary pulmonary aspergillosis (CPA) diagnosis with a) overall survival and comparison of survival with observed Kaplan-Meier estimates in this series: b) patients with/without pulmonary hypertension (PH) at CPA diagnosis, c) main pulmonary artery (MPA)/ ascending aorta diameter (AAD) ratio $>1$ or $\leqslant 1, d$ ) composite physiological index (CPI) score $>40$ or $\leqslant 40$, e) extent of fibrosis $>20 \%$ or $\leqslant 20 \%$ and $f$ ) predicted by the sarcoidosis staging model (Walsh algorithm [20]).

shown in two of our patients. Our series provides interesting information on risk factors for CPA in SA. Jobs with a high risk of mould exposure were more frequent in patients with CPA-SA than in HCs. Patients with CPA-SA were predominantly males, contrasting with our HC group and with the female predominance seen in advanced pulmonary SA [20]. No positive link was found with geographical origin, smoking habit, diabetes or treatment of SA. The male predominance might be due to more men in high-risk jobs such as construction work $[21,22]$. We agree with the recommendations of the Infectious Diseases Society of America, who "recommend reasonable precautions to reduce mould exposure among outpatients" with fibrocystic SA, "including avoidance of gardening, spreading mulch or close exposure to construction or renovation” [27].

Three deaths were due to massive haemoptysis. Threatening haemoptysis events were probably attributable to CPA, even though a bronchiectasis origin cannot definitely be ruled out. This rate (three patients being $11 \%$ of the deceased patients) was lower than the rate of $27 \%$ observed in a combined dated series $[5-8,15]$. Interventional radiology allowed immediate cessation of massive haemoptysis with often iterative interventions for bleeding relapse [28]. The literature is in favour of a reduction of death with interventional radiology in cases of threatening haemoptysis [29]. Only one of our patients benefited from intracavitary amphotericin B instillation, although this technique has been used in some series [30].

There are still few trials evaluating antifungals in CPA [31], and there is no study in the literature on CPA-SA. Our study shows that a short-term benefit can be achieved for CPA in SA with antifungals, as shown by CT according to the criteria of GODET et al. [23]. In fact, the maximal thickness of cavitation wall $(p=0.007)$ and pleura $(p=0.02)$ significantly decreased under antifungals. Complete recovery remained 
TABLE 6 Univariate analysis for survival at diagnosis of chronic pulmonary aspergillosis (CPA)

\begin{tabular}{lcccc} 
Parameter & Hazard ratio & Standard error & p-value & 95\% confidence interval \\
\hline Sex & 0.762 & 0.319 & 0.518 & $0.335-1.733$ \\
Geographical origin & 1.596 & 0.609 & 0.220 & $0.755-3.373$ \\
Age at sarcoidosis diagnosis & 1.030 & 0.016 & 0.065 & $0.998-1.062$ \\
Age at stage 4 diagnosis & 1.036 & 0.016 & 0.029 & $1.003-1.069$ \\
Age at CPA diagnosis & 1.054 & 0.017 & 0.002 & $1.020-1.090$ \\
Diabetes mellitus & 2.902 & 1.285 & 0.016 & $1.218-6.915$ \\
Extrapulmonary manifestations & 0.980 & 0.803 & 0.806 & $0.834-1.150$ \\
Pulmonary hypertension & 3.425 & 1.306 & 0.001 & $1.621-7.235$ \\
FEV & 0.979 & 0.011 & 0.069 & $0.958-1.001$ \\
FVC & 0.965 & 0.011 & 0.003 & $0.944-0.988$ \\
DLco & 0.968 & 0.010 & 0.003 & $0.947-0.988$ \\
CPI 40 & 0.293 & 0.147 & 0.015 & $0.109-0.784$ \\
MPA/AAD >1 & 2.347 & 1.020 & 0.050 & $1.001-5.501$ \\
CT fibrosis >20\% & 2.710 & 1.096 & 0.014 & $1.226-5.990$ \\
CT fibrosis extent & 1.041 & 0.012 & 0.001 & $1.017-1.066$ \\
Walsh's algorithm & 3.069 & 1.696 & 0.042 & $1.038-9.068$ \\
\hline
\end{tabular}

FEV1: forced expiratory volume in $1 \mathrm{~s}$; FVC: forced vital capacity; DLCo: diffusing capacity of the lung for carbon monoxide; CPI: composite physiological index; MPA/AAD: main pulmonary artery/ascending aorta diameter ratio; CT: computed tomography.

exceptional and probably required both CPA control and cavitation reversibility, a condition illustrated in an exceptional case in figure 3.

Controversy remains regarding surgical CPA care [32], as peri-operative management of CPA-SA is challenging [6]. A later aspergillosis relapse occurred 6-45 months after surgery in all cases except one. Resection surgery should be proposed in strictly localised aspergillomas [33], a very rare condition in CPA-SA, and for severe bleeding that is best controlled by BAE, as recommended [27].

This study, being retrospective and monocentric, has some limitations. A prospective systematic recording of domestic and environmental mould exposure was not available in files, unlike past and current occupational exposure.

\section{Conclusion}

In CPA-SA, mortality appeared to be conditioned mainly by the severity of the underlying pulmonary SA rather than by CPA. Improvement in CPA-SA care should probably include measures preventing exposure to spores for fibrocystic SA, effective antifibrotic drugs for patients with a poor prognosis, and more effective antifungal drugs.

Acknowledgements

We thank Jean-Paul Battesti, Christine Person, Bruno Philippe, Stephane Dominique, Emmanuel Bergot, Elisabeth Rivaud and Pascaline Priou.

\section{References}

1 Denning DW, Cadranel J, Beigelman-Aubry C, et al. Chronic pulmonary aspergillosis: rationale and clinical guidelines for diagnosis and management. Eur Respir J 2016; 47: 45-68.

2 Denning DW, Pleuvry A, Cole DC. Global burden of chronic pulmonary aspergillosis complicating sarcoidosis Eur Respir J 2013; 41: 621-626.

3 Smith NL, Denning DW. Underlying conditions in chronic pulmonary aspergillosis including simple aspergilloma. Eur Respir J 2011; 37: 865-872.

4 Sones M, Israel HL. Course and prognosis of sarcoidosis. Am J Med 1960; 29: 84-93.

5 Kaplan J, Johns CJ. Mycetomas in pulmonary sarcoidosis: non-surgical management. Johns Hopkins Med J 1979; 145: $157-161$.

6 Israel HL, Lenchner GS, Atkinson GW. Sarcoidosis and aspergilloma. The role of surgery. Chest 1982; 82: 430-432.

7 Wollschlager C, Khan F. Aspergillomas complicating sarcoidosis. A prospective study in 100 patients. Chest 1984; 86: 585-588.

8 Tomlinson JR, Sahn SA. Aspergilloma in sarcoid and tuberculosis. Chest 1987; 92: 505-508.

9 Jewkes J, Kay PH, Paneth M, et al. Pulmonary aspergilloma: analysis of prognosis in relation to haemoptysis and survey of treatment. Thorax 1983; 38: 572-578.

10 Nam HS, Jeon K, Um SW, et al. Clinical characteristics and treatment outcomes of chronic necrotizing pulmonary aspergillosis: a review of 43 cases. Int J Infect Dis 2010; 14: e479-e482. 
11 Pena TA, Soubani AO, Samavati L. Aspergillus lung disease in patients with sarcoidosis: a case series and review of the literature. Lung 2011; 189: 167-172.

12 Nardi A, Brillet PY, Letoumelin P, et al. Stage IV sarcoidosis: comparison of survival with the general population and causes of death. Eur Respir J 2011; 38: 1368-1373.

13 Shlobin OA, Nathan SD. Management of end-stage sarcoidosis: pulmonary hypertension and lung transplantation. Eur Respir J 2012; 39: 1520-1533.

14 Statement on sarcoidosis. Joint Statement of the American Thoracic Society (ATS), the European Respiratory Society (ERS) and the World Association of Sarcoidosis and Other Granulomatous Disorders (WASOG) adopted by the ATS Board of Directors and by the ERS Executive Committee, February 1999. Am J Respir Crit Care Med 1999; 160: 736-755.

15 Judson MA, Costabel U, Drent M, et al. The WASOG Sarcoidosis Organ Assessment Instrument: an update of a previous clinical tool. Sarcoidosis Vasc Diffuse Lung Dis 2014; 31: 19-27.

16 Cotes JE, Chinn DJ, Quanjer PH, et al. Standardization of the measurement of transfer factor (diffusing capacity). Eur Respir J 1993; 6: Suppl. 16, 41-52.

17 Quanjer PH, Tammeling GJ, Cotes JE, et al. Lung volumes and forced ventilatory flows. Eur Respir J 1993; 6: Suppl. 16, 5-40.

18 Abehsera M, Valeyre D, Grenier P, et al. Sarcoidosis with pulmonary fibrosis: CT patterns and correlation with pulmonary function. AJR Am J Roentgenol 2000; 174: 1751-1757.

19 Wells AU, Desai SR, Rubens MB, et al. Idiopathic pulmonary fibrosis: a composite physiologic index derived from disease extent observed by computed tomography. Am J Respir Crit Care Med 2003; 167: 962-969.

20 Walsh SL, Wells AU, Sverzellati N, et al. An integrated clinicoradiological staging system for pulmonary sarcoidosis: a case-cohort study. Lancet Respir Med 2014; 2: 123-130.

21 Caira M, Candoni A, Verga L, et al. Pre-chemotherapy risk factors for invasive fungal diseases: prospective analysis of 1,192 patients with newly diagnosed acute myeloid leukemia (SEIFEM 2010-a multicenter study). Haematologica 2015; 100: 284-292.

22 Sipsas NV, Kontoyiannis DP. Occupation, lifestyle, diet, and invasive fungal infections. Infection 2008; 36: 515-525.

23 Godet C, Laurent F, Bergeron A, et al. Computed tomography assessment of response to treatment in chronic pulmonary aspergillosis. Chest 2016; 150: 139-147.

24 Aspergilloma and residual tuberculous cavities-the results of a resurvey. Tubercle 1970; 51: $227-245$.

25 Keir GJ, Garfield B, Hansell DM, et al. Cyclical caspofungin for chronic pulmonary aspergillosis in sarcoidosis. Thorax 2014; 69: 287-288.

26 Guleria R, Pande JN. Endoscopic instillation of fungicide to treat aspergilloma. Lancet 1996; $348: 621$.

27 Patterson TF, Thompson GR 3rd, Denning DW, et al. Practice Guidelines for the Diagnosis and Management of Aspergillosis: 2016 update by the Infectious Diseases Society of America. Clin Infect Dis 2016; 63: e1-e60.

28 Fartoukh M, Parrot A, Khalil A. Aetiology, diagnosis and management of infective causes of severe haemoptysis in intensive care units. Curr Opin Pulm Med 2008; 14: 195-202.

29 Fartoukh M, Khoshnood B, Parrot A, et al. Early prediction of in-hospital mortality of patients with hemoptysis: an approach to defining severe hemoptysis. Respiration 2012; 83: 106-114.

30 Kravitz JN, Berry MW, Schabel SI, et al. A modern series of percutaneous intracavitary instillation of amphotericin B for the treatment of severe hemoptysis from pulmonary aspergilloma. Chest 2013; 143: 1414-1421.

31 Agarwal R, Vishwanath G, Aggarwal AN, et al. Itraconazole in chronic cavitary pulmonary aspergillosis: a randomised controlled trial and systematic review of literature. Mycoses 2013; 56: 559-570.

32 Farid S, Mohamed S, Devbhandari M, et al. Results of surgery for chronic pulmonary Aspergillosis, optimal antifungal therapy and proposed high risk factors for recurrence-a National Centre's experience. J Cardiothorac Surg 2013; 8: 180 .

33 Lejay A, Falcoz PE, Santelmo N, et al. Surgery for aspergilloma: time trend towards improved results? Interact Cardiovasc Thorac Surg 2011; 13: 392-395. 